\section{Características das vozes roucas, ásperas e normais: análise acústica espectrográfica comparativa}

\author{
Paulo A. L. Pontes ${ }^{1}$, Vanessa P. Vieira ${ }^{2}$, \\ Maria I. R. Gonçalves ${ }^{3}$, Antônio A. L. Pontes ${ }^{4}$
}

\section{Characteristics of hoarse, rough and normal voices: acoustic spectrographic comparative analysis}

Palavras-chave: espectrografia, rouquidão, qualidade vocal. Key words: spectrography, hoarseness, vocal quality.

Resumo / Summary

I ntrodução: A avaliação da qualidade vocal pode ser realizada pelos métodos perceptivo auditivo e acústico, e não existem termos padronizados para a sua descrição. Entretanto, dois desses termos merecem atenção quanto aos parâmetros que os distinguem: a aspereza e a rouquidão, ambos denominados genericamente de rouquidão na literatura. Objetivo: Avaliar comparativamente vozes roucas, ásperas e normais por meio da espectrografia. Forma de estudo: Prospectivo clínico randomizado. Material e método: Seis vozes roucas, seis ásperas e seis normais foram selecionadas por meio da avaliação perceptivo auditiva, e analisadas espectrograficamente sob cinco parâmetros: análise da freqüência fundamental $\left(\mathrm{f}_{0}\right)$, extensão dos harmônicos, definição de harmônicos, presença de ruído entre os harmônicos e acima deles. Resultados: As vozes roucas apresentaram $\mathrm{f}_{0}$ mais grave e as ásperas mais aguda quando comparadas com as vozes normais. Considerando o espectrograma de 0 a $5,4 \mathrm{kHz}$, o valor médio da freqüência que o harmônico mais alto alcançou foi $4.555 \mathrm{~Hz}$ para vozes normais, $2.125 \mathrm{~Hz}$ para vozes ásperas e $1.147 \mathrm{~Hz}$ para vozes roucas. O traçado dos harmônicos nas vozes roucas apresentou qualidade de definição inferior aos outros dois tipos de vozes e maior quantidade de ruído entre e acima dos harmônicos. Conclusões: Existem diferenças espectrográficas marcantes entre essas vozes. Os harmônicos estão presentes em grande quantidade e com melhor definição nas vozes normais, e decrescem em quantidade e qualidade nas vozes ásperas e roucas, respectivamente. Inversamente, o ruído está presente em grande quantidade e de modo difuso nas vozes roucas e em menor quantidade nas vozes ásperas e normais.

\section{工}

ntroduction: Vocal quality can be assessed by auditory perceptual and acoustic methods. The terms used to describe vocal quality aren't standardized. However, two of them deserve special attention regarding the parameters that distinguish roughness and hoarseness, both usually called as hoarseness in the literature. Aim: To evaluate comparatively and to define basic differences among hoarse, rough and normal voices using acoustic spectrography. Study Design: Prospective clinical randomized. Material and Method: Six hoarse, six rough and six normal voices sorted by auditory perceptual evaluation, and analyzed by spectrography under five parameters: fundamental frequency $\left(\mathrm{f}_{0}\right)$, extension of harmonics, definition of harmonics, presence of noise between the harmonics and up them. Results: Hoarse voices presented lower $\mathrm{f}_{0}$, whereas rough voices showed higher $\mathrm{f}_{0}$ in comparison to normal voices. Considering the spectrogram from 0 to $5,4 \mathrm{kHz}$, the mean value of the uppermost harmonic frequency reached 4.555 $\mathrm{Hz}$ for normal voices, $2.125 \mathrm{~Hz}$ for rough voices and 1.147 $\mathrm{Hz}$ for hoarse voices. Harmonic tracing in hoarse voices showed worse definition and greater amount of noise among and up the harmonics. Conclusions: There are striking spectrographic differences among hoarse, rough and normal voices. Harmonics are present in greater amount and show better definition in normal voices, and decrease in amount in rough and hoarse voices respectively. Inversely, noise appears in great amount and in a diffuse way in hoarse voices and in smaller amount in rough and normal voices.

\footnotetext{
${ }^{1}$ Professor titular de Otorrinolaringologia do Departamento de Otorrinolaringologia e Distúrbios da Comunicação Humana da Universidade Federal de São Paulo - UNIFESP - EPM. ${ }^{2}$ Fga. Especialização em Distúrbios da Comunicação Humana pela Universidade Federal de São Paulo - UNIFESP- EPM. Professora do curso de Fonoaudiologia da Universidade Federal de São Paulo - EPM. ${ }^{4}$ Médico do Instituto da Laringe - São Paulo.
}

Instituto da Laringe - INLAR - São Paulo - Rua Dr. Diogo de Faria, 171 Vila Clementino - São Paulo - Brasil CEP 04037-000 Telefone: (0xx11)5549.2188 - Fax (0xx11) 5575.7649 - E-mail: vpedrosa@inlar.com.br

Trabalho apresentado no II Congresso Triológico de Otorrinolaringologia da Cidade de Goiânia. Agosto de 2001. Artigo recebido em 24 de janeiro de 2002. Artigo aceito em 21 de março de 2002. 


\section{INTRODUÇÃO}

Na avaliação da qualidade vocal pode-se utilizar dois recursos importantes: a análise perceptivo-auditiva e a análise acústica.

A primeira é um método subjetivo que varia de acordo com o avaliador, seus conceitos pessoais sobre a qualidade vocal, habilidades de percepção, discriminação e experiência.

A segunda análise é objetiva e faz uso de programas computadorizados que demonstram quantitativamente vários aspectos mensuráveis do sinal de voz captado. A análise acústica alcançou maior utilização na última década, principalmente no Brasil, pois nos últimos anos os estudos nesta área se tornaram mais abrangentes. Ela permite a avaliação objetiva da vOz em situações iniciais e seguimento dos tratamentos fonoaudiológicos, bem como no pré- e pós-operatório e na investigação científica. Uma das principais ferramentas desta análise é a espectrografia, que demonstra visualmente as características acústicas da emissão e complementa a avaliação perceptivo-auditiva. No entanto, as informações apresentadas por ela exigem interpretação por parte do avaliador.

A espectrografia é a tradução de padrões sonoros em padrões visuais gráficos que refletem a freqüência fundamental $\left(f_{0}\right)$ e os harmônicos de uma voz, bem como as zonas de forte intensidade dos sons da fala, utilizando uma análise matemática denominada "análise de Fourier", que determina os componentes da onda sonora. Este método faz a dissecação da onda acústica em seus componentes básicos, ou seja, expõe as freqüências e as respectivas amplitudes que a constitui ${ }^{1}$. Desta forma, o espectrograma pode ser definido como um gráfico que mostra as intensidades relativas dos componentes das ondas sonoras, indicadas pelo escurecimento ou coloração de faixas de freqüência no eixo vertical em função do tempo no eixo horizontal. Nesta avaliação o especialista poderá inferir a função glótica durante a emissão vocal. Nos dias atuais esta correlação é facilitada pela captação de imagens laríngeas com o recurso de fibras ópticas.

A terminologia relacionada à classificação da qualidade vocal é muito variada e ambígüa. Existem poucos termos padronizados e, normalmente, os avaliadores para compreenderem melhor as características do som ouvido, utilizam adjetivos relacionados aos órgãos dos sentidos como: voz clara, rugosa, forçada, escura, vibrante, pálida, ácida, entre outros. Dentre os vários tipos de qualidades de vozes consideradas alteradas duas merecem atenção quanto aos seus parâmetros distintivos: a voz rouca e a voz áspera, ambas chamadas genericamente de rouquidão pela literatura internacional.

Vários autores fazem referência à aspereza, rouquidão e disfonia de forma indefinida, como sendo uma mesma alteração vocal ${ }^{2,3,4,5}$.
$\mathrm{Na}$ língua portuguesa, apesar de toda confusão e polêmica terminológica relacionada à qualidade vocal, do ponto de vista conceitual é imperioso fazer a distinção entre as vozes rouca e áspera.

A voz rouca possui característica ruidosa, com altura e intensidade freqüentemente diminuídas, enquanto que na voz áspera o som provoca uma sensação desagradável e até mesmo irritante ${ }^{6}$. A voz áspera também é definida como uma impressão psicoacústica da irregularidade de vibração das pregas vocais, isto é, corresponde a flutuações irregulares na freqüência fundamental e/ou na amplitude da fonte sonora glótica².

Quanto à voz normal, não existem definições exatas ou aceitáveis sobre suas características. Segundo Casper e Colton ${ }^{7}$, não há padrões nem limites definidos a este respeito. Greene e Mathieson ${ }^{8}$ a caracterizam como uma voz comum, que não apresenta nada especial em seu som. Moore 9 afirmou que o critério de separar as vozes em normais e alteradas é determinado pelos ouvintes, sendo que as desordens vocais são culturalmente baseadas e socialmente determinadas.

Estes conceitos deverão ter uma tradução espectrográfica diferenciada, pois os autores ao procurarem identificar parâmetros que definam rouquidão, não deixam claras as diferenças entre esses dois tipos de vozes.

Assim, o objetivo deste trabalho é analisar comparativamente, por meio da espectrografia, vozes roucas, ásperas e normais previamente selecionadas pelo método perceptivo-auditivo.

\section{MATERIAL E MÉTODO}

Foram estudados dezoito adultos divididos em 3 grupos: vozes normais, roucas e ásperas.ada grupo era composto por 3 adultos do sexo masculino e 3 do sexo feminino; os três grupos tiveram limites de idade entre vinte e 45 anos. A média de idade entre os homens com vozes roucas foi de 35 anos, com vozes ásperas de 34,6 anos e com vozes normais foi de 30 anos. No grupo feminino a média entre as mulheres com vozes roucas foi de 39,6 anos, com vozes ásperas foi de 37 anos e com vozes normais de 34 anos (Quadro 1).

Os casos foram selecionados de gravações de vídeo de exames laringológicos, por telescopia rígida realizados no Instituto da Laringe - INLAR, São Paulo. O trabalho foi submetido à aprovação do Comitê de Ética em Pesquisa da Universidade Federal de São Paulo - UNIFESP/EPM.

Os equipamentos utilizados para estes exames foram: Microfone multidirecional; Fita de gravação digital marca SONY 60 LP-90; Vídeo cassete digital marca SONY modelo DHR 100. A gravação do exame foi realizada em ambiente silencioso, com um distanciamento bocamicrofone de $15 \mathrm{~cm}$, durante a emissão da vogal $/ \varepsilon /$ sustentada em condição habitual. A seleção das vozes 
foi realizada por duas fonoaudiólogas e dois otorrinolaringologistas com experiência nesta avaliação. O critério de seleção para vozes alteradas foi, além da presença de voz rouca e voz áspera, apresentarem grau 3 de comprometimento numa avaliação de intensidade de 1 a 3, sendo 1-leve, 2-moderado e 3-extremo. Já os indivíduos com vozes normais não apresentavam queixas vocais e apresentavam voz "limpa", sem alterações. Como definição de voz rouca considerou-se uma voz ruidosa, que contrasta com a suavidade e a sensação de harmonia da voz normal. Neste tipo de voz, a altura e a intensidade estão freqüentemente diminuídas com a presença de ruídos. Como áspera consideramos a voz com uma característica rude, pouco melodiosa, desagradável e até mesmo irritante da emissão e com variação do $p i t c b^{6}$. Como voz normal selecionamos vozes com qualidade aceitável socialmente, sem interferência na inteligibilidade da fala e apresentando freqüência, intensidade, modulação e projeção apropriadas para o sexo e idade do falante ${ }^{10}$. Não houve discordância entre os ouvintes durante a seleção das emissões.

Após a coleta e seleção, as vozes foram transferidas para um computador PC compatível com Macintosh, com sistema 7.5, utilizando o programa SoundScope desenvolvido pela $\mathrm{GW}$ instruments.

O programa SoundScope gera uma saída de dados em forma de espectrograma. Para a realização desta avaliação foi utilizado tempo de emissão da voz sustentada de um segundo, faixa de freqüência de $0,0 \mathrm{kHz}$ a $5,4 \mathrm{kHz}$ no espectrograma e amplitude de 4,0 V no osciloscópio. No espectrograma foi utilizado um filtro passa-faixa de 59 $\mathrm{Hz}$ - banda estreita.

Quadro 1. Distribuição dos casos de acordo com o sexo, idade e qualidade vocal classificadas pelo método perceptivoauditivo

\begin{tabular}{|cccc|}
\hline Caso & Idade & Sexo & Qualidade vocal \\
\hline 01 & 30 & Feminino & Áspera \\
02 & 43 & Masculino & Áspera \\
03 & 40 & Masculino & Rouca \\
04 & 30 & Masculino & Áspera \\
05 & 20 & Masculino & Rouca \\
06 & 45 & Feminino & Áspera \\
07 & 31 & Masculino & Áspera \\
08 & 36 & Feminino & Áspera \\
09 & 45 & Masculino & Rouca \\
10 & 30 & Feminino & Rouca \\
11 & 45 & Feminino & Rouca \\
12 & 44 & Feminino & Rouca \\
13 & 20 & Feminino & Normal \\
14 & 38 & Feminino & Normal \\
15 & 44 & Feminino & Normal \\
16 & 30 & Masculino & Normal \\
17 & 23 & Masculino & Normal \\
18 & 37 & Masculino & Normal \\
\hline
\end{tabular}

Para algumas vozes foi necessário modificar o ganho no próprio software para que as intensidades ficassem sempre dentro do limite pré-estabelecido de 4,0 V. Esta modificação teve por finalidade padronizar a intensidade nos espectrogramas permitindo a análise comparativa entre harmônicos e ruídos. Dessa forma, evitou-se a padronização da intensidade durante a emissão que poderia provocar a modificação no gesto fonatório.

Para este estudo foram utilizados parâmetros distintivos relativos à onda sonora bem como os relativos ao ruído, como componentes principais da qualidade vocal.

Parâmetros relativos à onda sonora:

- Análise computadorizada da $\mathrm{f}_{0}$;

- Extensão dos harmônicos: freqüências cujos harmônicos mais altos se apresentam de forma definida sem interrupções; a freqüência do harmônico superior foi definida como o múltiplo da $\mathrm{f}_{0}$, que coincide com a região do mesmo no espectro;

- Definição dos harmônicos.

Parâmetros relativos ao ruído:

- Presença de ruído na região dos harmônicos;

- Presença de ruído na região acima dos harmônicos.

Os resultados foram submetidos à análise estatística simples de porcentagem e média aritmética.

\section{RESULTADOS}

Os resultados estão dispostos nas Tabelas de 1 a 5 .

$\mathrm{Na}$ Tabela 1 estão os resultados da análise computadorizada da $\mathrm{f}_{0}$. Nela pode-se observar que nas vozes roucas a $\mathrm{f}_{0}$ mostrou-se muito grave, enquanto que nas ásperas os resultados foram muito altos, ou seja, agudos quando comparados com as vozes normais e comparadas entre os sexos.

Quanto aos valores das freqüências dos harmônicos superiores, dispostos na Tabela 2 , as vozes roucas apresentaram médias baixas quando comparadas com as vozes ásperas em relação ao sexo, e ambas apresentaram um alcance muito baixo em relação às vozes normais.

$\mathrm{Na}$ análise da definição dos traçados dos harmônicos (Tabela 3) todas as vozes roucas apresentaram pior qualidade de definição que as vozes normais e ásperas.

$\mathrm{Na}$ Tabela 4, em relação à intensidade do ruído entre os harmônicos, as vozes ásperas apresentaram menor quantidade de ruído que as vozes roucas, ou seja, esta região é menos acinzentada nas vozes ásperas do que nas vozes roucas.

Finalmente, a Tabela 5 mostra a quantidade de ruído na região acima da faixa dos harmônicos e seus resultados foram semelhantes aos da Tabela anterior, pois as vozes roucas também apresentaram mais ruído nesta região que as vozes ásperas. 
Tabela 1. Valores das freqüências fundamentais e respectivas médias, em Hz, nos sexos masculino e feminino, para cada indivíduo dos três grupos de vozes

\begin{tabular}{|c|c|c|c|c|c|c|}
\hline \multicolumn{3}{|c|}{ Rouca } & \multicolumn{2}{|c|}{ Áspera } & \multicolumn{2}{|c|}{ Normal } \\
\hline & Masculina & Feminina & Masculina & Feminina & Masculina & Feminina \\
\hline & 70 & 137 & 199 & 181 & 102 & 198 \\
\hline & 133 & 161 & 186 & 315 & 122 & 229 \\
\hline & 117 & 111 & 166 & 206 & 142 & 232 \\
\hline$\overline{\bar{X}}$ & 106,6 & 136,3 & 183,6 & 234,0 & 122,0 & 219,6 \\
\hline
\end{tabular}

Tabela 3. Distribuição dos casos em ordem crescente de definição do traçado dos harmônicos, especificando a qualidade vocal e sexo

\begin{tabular}{ccc}
\hline Caso & Qualidade vocal & Sexo \\
\hline 12 & Rouca & Feminino \\
10 & Rouca & Feminino \\
03 & Rouca & Masculino \\
09 & Rouca & Masculino \\
05 & Rouca & Masculino \\
11 & Rouca & Feminino \\
01 & Áspera & Feminino \\
06 & Áspera & Feminino \\
02 & Áspera & Masculino \\
07 & Áspera & Masculino \\
04 & Áspera & Masculino \\
08 & Áspera & Feminino \\
18 & Normal & Masculino \\
17 & Normal & Masculino \\
16 & Normal & Masculino \\
13 & Normal & Feminino \\
15 & Normal & Feminino \\
14 & Normal & Feminino \\
\hline
\end{tabular}

Tabela 5. Distribuição dos casos em ordem crescente de intensidade de ruído na região acima dos harmônicos, especificando a qualidade vocal e o sexo

\begin{tabular}{ccc}
\hline Caso & Qualidade vocal & Sexo \\
\hline $13^{*}$ & Normal & Feminino \\
15 & Normal & Feminino \\
14 & Normal & Feminino \\
$17^{*}$ & Normal & Masculino \\
$18^{*}$ & Normal & Masculino \\
16 & Normal & Masculino \\
04 & Áspera & Masculino \\
02 & Áspera & Masculino \\
01 & Áspera & Feminino \\
08 & Áspera & Feminino \\
06 & Áspera & Feminino \\
07 & Áspera & Masculino \\
10 & Rouca & Feminino \\
11 & Rouca & Masculino \\
09 & Rouca & Masculino \\
05 & Rouca & Masculino \\
03 & Rouca & Masculino \\
12 & Rouca & Feminino \\
\hline
\end{tabular}

* Vozes normais que apresentaram o harmônico superior abaixo do limite do espectrograma.
Tabela 2. Valores dos harmônicos superiores na espectrografia e as respectivas médias, em $\mathrm{Hz}$, nos sexos masculino e feminino, dos três grupos de vozes

\begin{tabular}{ccccccccc}
\hline & \multicolumn{2}{c}{ Rouca } & & \multicolumn{2}{c}{ Áspera } & & \multicolumn{2}{c}{ Normal } \\
\cline { 2 - 3 } \cline { 7 - 8 } Masculina & Feminina & & Masculina & Feminina & & Masculina & Feminina \\
\hline & 700 & 2192 & & 1592 & 2172 & & 3570 & 5148 \\
& 1197 & 966 & & 2232 & 2205 & & 3904 & 4122 \\
& 1053 & 777 & & 2490 & 2060 & & 5254 & 5336 \\
\hline $\bar{X}$ & 983,3 & 1311,6 & 2104,6 & 2145,6 & & 4242,6 & 4868,6 \\
\hline
\end{tabular}

Tabela 4. Distribuição dos casos em ordem crescente de intensidade ruído na região entre os harmônicos, especificando a qualidade vocal e o sexo

\begin{tabular}{ccc}
\hline Caso & Qualidade vocal & Sexo \\
\hline 15 & Normal & Feminino \\
13 & Normal & Feminino \\
14 & Normal & Feminino \\
16 & Normal & Masculino \\
18 & Normal & Masculino \\
17 & Normal & Masculino \\
01 & Áspera & Feminino \\
06 & Áspera & Feminino \\
02 & Áspera & Masculino \\
08 & Áspera & Feminino \\
07 & Áspera & Masculino \\
04 & Áspera & Masculino \\
10 & Rouca & Feminino \\
11 & Rouca & Feminino \\
09 & Rouca & Masculino \\
03 & Rouca & Masculino \\
05 & Rouca & Masculino \\
12 & Rouca & Feminino \\
\hline
\end{tabular}

\section{DISCUSSÃO}

Os termos usados para qualificar a voz variam quanto à língua e à cultura, não apenas nas palavras, mas também no campo semântico. Cada idioma possui adjetivos para descrever a qualidade vocal normal e alterada.

Neste trabalho, dentre as vozes alteradas, foram escolhidos dois grupos perceptivamente diferentes $\mathrm{e}$ denominados de vozes roucas e ásperas, segundo Behlau e Pontes ${ }^{6}$. Foi usada a definição de Behlau et $\mathrm{al}^{10}$ para a seleção das vozes normais.

Foram observados harmônicos em todos os espectrogramas, sendo que nas vozes roucas eles apresentaram-se em menor número e com um traçado menos definido, devido à presença de ruído e talvez da variação da $\mathrm{f}_{0}$. Apenas em uma das vozes femininas é que se pôde observar uma melhor definição dos harmônicos, mas com muito escurecimento da região entre eles. O estudo de Hiraoka et $\mathrm{al}^{11}$ também mostrou, acusticamente, a diferença entre vozes roucas e normais através da intensidade dos harmônicos. 
CASO 8

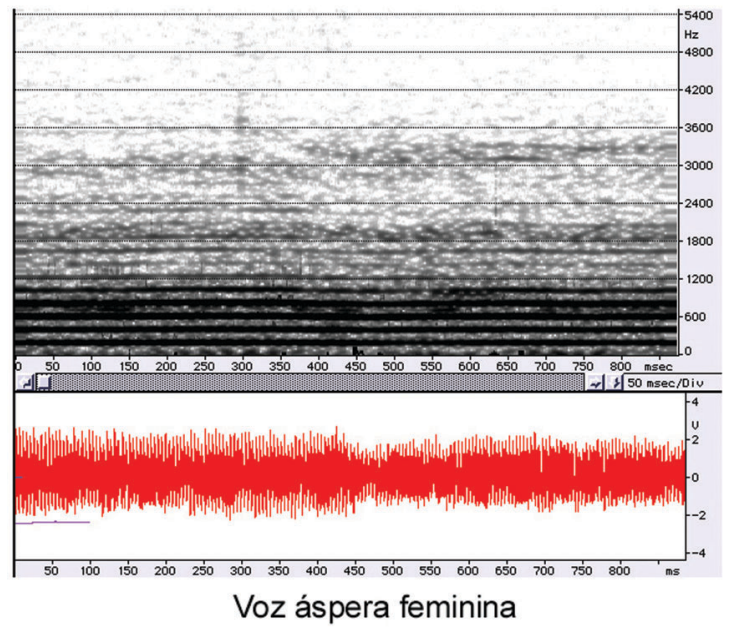

CASO 15

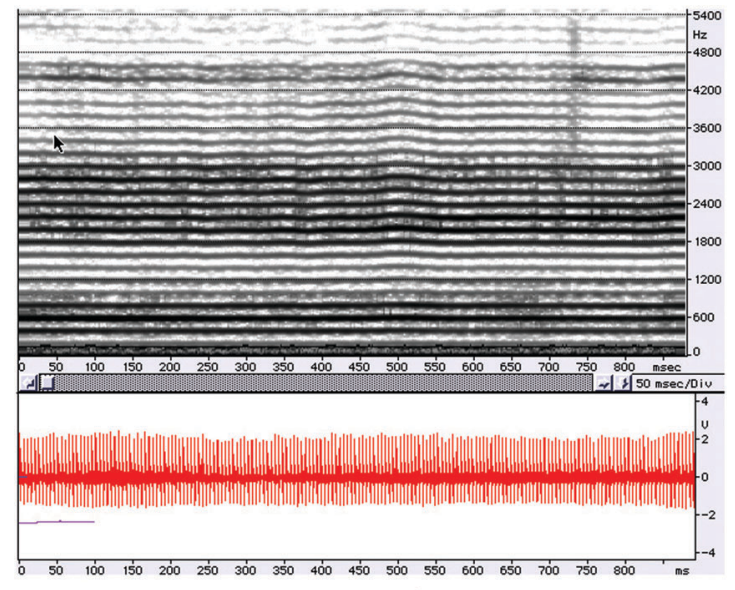

Voz normal feminina

\section{CASO 11}

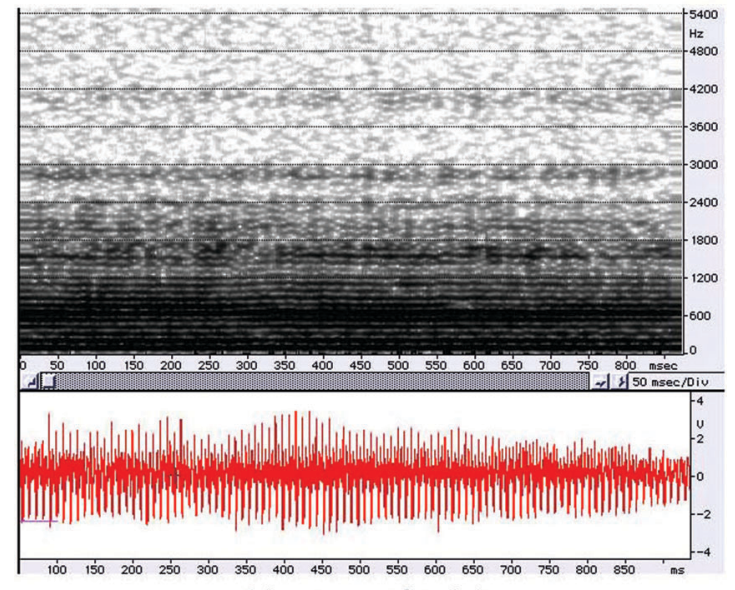

Voz rouca feminina

\section{CASO 4}

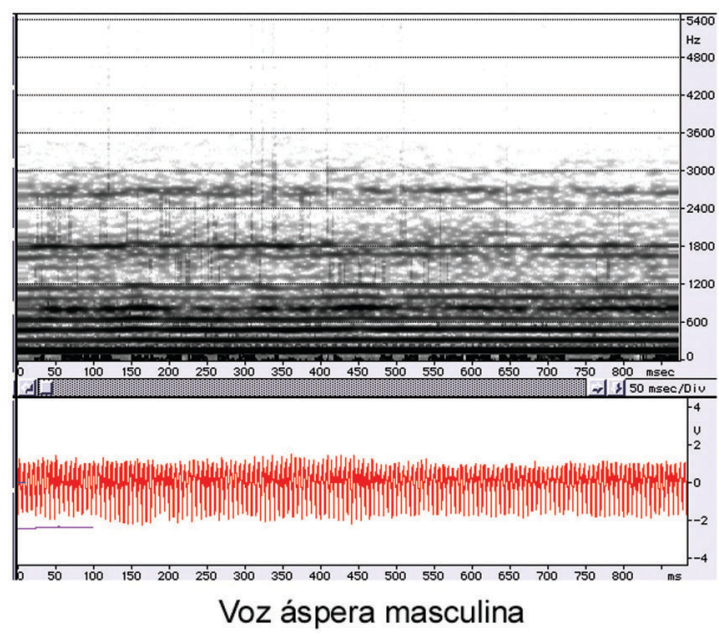

CASO 17

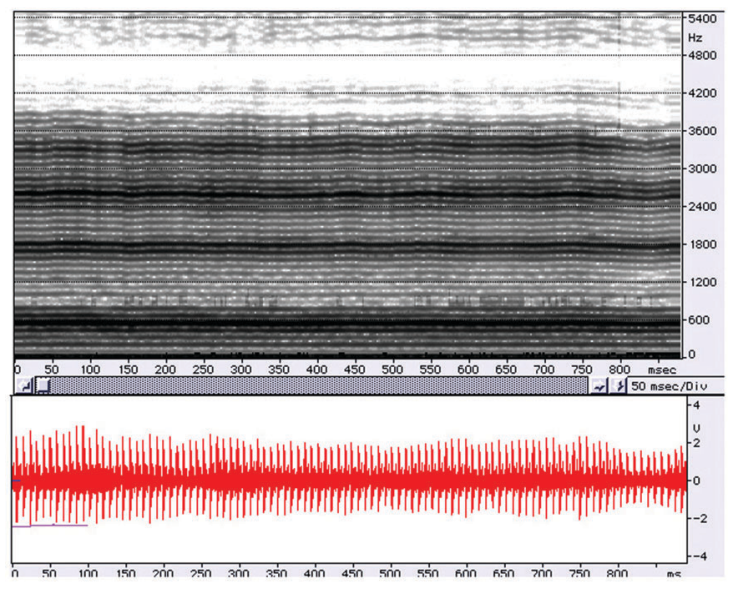

Voz normal masculina

\section{CASO 5}

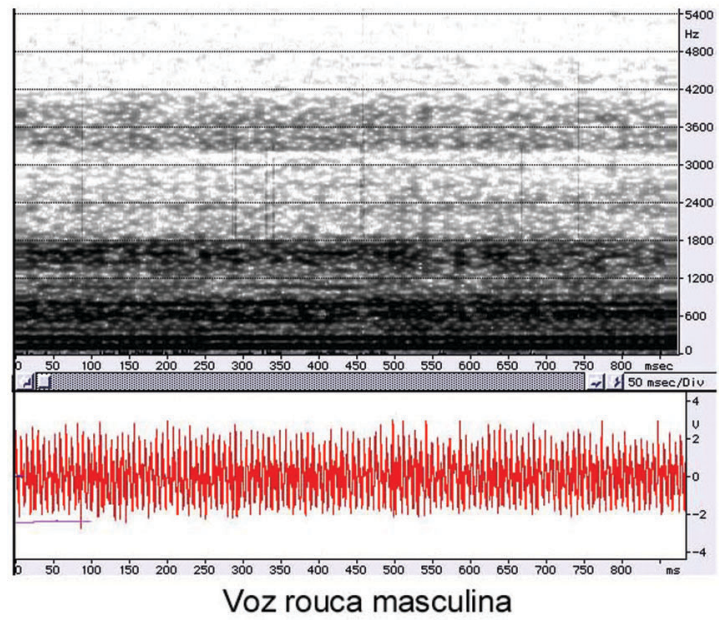


Quanto à $\mathrm{f}_{0}$ (Tabela 1) no sexo masculino, a média normal esperada é $113 \mathrm{~Hz}^{12}$; no entanto encontramos 183,6 $\mathrm{Hz}$ nas vozes ásperas masculinas, ou seja, valor bem acima do normal e, nas vozes roucas houve um rebaixamento discreto da média para 106,6 Hz. Inversamente, na mulher o rebaixamento da $\mathrm{f}_{0}$ para $136,3 \mathrm{~Hz}$ nas vozes roucas foi bastante acentuado em relação ao normal esperado de $207 \mathrm{~Hz}^{12}$, enquanto que a $\mathrm{f}_{0}$ das vozes ásperas elevou-se para $234,0 \mathrm{~Hz}$. Em outras palavras, a voz rouca apresentou $\mathrm{f}_{0}$ mais grave que o normal e a áspera mais aguda. Podese também afirmar por estes resultados que a elevação da $\mathrm{f}_{0}$ na voz áspera no sexo masculino é tão marcante quanto o rebaixamento para a voz rouca no sexo feminino. Este comportamento pode ser justificado admitindo que, no espectro da conversação, a vOz feminina e a masculina ocupam posições opostas na gama de freqüências das vozes, sendo, portanto, mais fáceis as variações dentro desta gama do que além dos seus limites. Arnold e Emanuel ${ }^{13}$, em seu estudo sobre a percepção da aspereza em vogais sustentadas, também encontraram a $\mathrm{f}_{0}$ aumentada em 4 de 10 sujeitos com vozes ásperas.

Nas vozes normais a média da $\mathrm{f}_{0}$ na população masculina foi de $122,0 \mathrm{~Hz}$, estando próxima da média obtida no estudo de Behlau et al ${ }^{12}$ de $113,0 \mathrm{~Hz}$, com um desvio padrão de $6,26 \mathrm{~Hz}$. Também para o sexo feminino a média obtida de $219,6 \mathrm{~Hz}$ está próxima ao valor encontrado no mesmo estudo de $207,8 \mathrm{~Hz}$ com um desvio padrão de $4,49 \mathrm{~Hz}$.

Na Tabela 2 pode ser observado que para os casos normais os harmônicos preenchem todo ou quase todo o intervalo do espectrograma com uma média de alcance nas vozes femininas de $4.868,6 \mathrm{~Hz}$ e nas masculinas de 4.242,6 Hz. Essa riqueza de harmônicos exprime maior clareza vocal. Já nas vozes ásperas estas faixas alcançaram a média de $2.145,6 \mathrm{~Hz}$ no sexo feminino e no masculino de $2.104,6 \mathrm{~Hz}$, representando praticamente a metade da média dos normais; nos roucos a média superior foi de 1.311,6 Hz para os casos de vozes femininas e de 983,3 $\mathrm{Hz}$ para as masculinas, representando quase que um quarto do resultado das vozes normais. Essa pobreza de harmônicos pode ser explicada pelo teorema de Fourier. Por este teorema, pode-se supor que a perda de harmônicos nas freqüências altas na voz áspera é a conseqüência de ciclos vibratórios com diferentes períodos e intensidades; os harmônicos inferiores estão com muita intensidade (energia acústica) no espectrograma, dando a configuração da linha horizontal no eixo tempo; já nos harmônicos superiores, devido à aperiodicidade dos ciclos vibratórios, o harmônico se distancia a cada ciclo com a perda de intensidade, o que torna menos definidos os harmônicos superiores, ou seja, os harmônicos deixam de ser observados em relação ao eixo tempo por estarem em níveis distintos no eixo das freqüências. Já nas vozes roucas, não se tem somente um tom fundamental aperiódico, mas sim uma somatória de sons e, por este motivo, a definição dos harmônicos foi menor e o alcance dos mesmos bem mais baixo.

Da mesma forma pode-se explicar a configuração dos harmônicos na Tabela 3; nas vozes roucas estes aparecem muito mal definidos, com instabilidade e baixa amplitude na espectrografia e, nas vozes ásperas, a definição é moderada e o escurecimento entre eles é mais homogêneo, como observado também na Tabela 4. Este escurecimento nas vozes ásperas pode ser explicado de acordo com os relatos de Omori et $\mathrm{al}^{4}$, que definiram a presença de diferentes anormalidades acústicas em algumas vozes alteradas. Eles documentaram na potência espectral baixos picos entre dois harmônicos consecutivos. Estes picos são denominados subharmônicos, que podem ser considerados, neste material, como a faixa cinzenta entre cada harmônico representado na espectrografia das vozes ásperas.

No mesmo sentido, para Nunez et $\mathrm{al}^{14}$, subharmônicos são modulações de baixa freqüência localizados entre dois harmônicos consecutivos no espectro. Deal e Emanuel ${ }^{15}$, em seu estudo sobre a forma de onda e o traço espectral de vozes ásperas, concluíram que uma maior variação da amplitude permite uma melhor percepção auditiva da aspereza. Também Emanuel e Austin ${ }^{16}$ selecionaram 20 indivíduos com vozes normais e ásperas, realizaram a análise acústica e concluíram que os valores obtidos diferenciam significativamente as vozes perceptivamente normal e áspera.

Por outro lado, nas vozes roucas, como geralmente não se tem uma boa definição dos harmônicos, as faixas acinzentadas são, provavelmente, apenas ruídos a eles sobrepostos (Tabela 4), sendo intensa e difusa a presença destes entre os harmônicos nestes casos.

Nas Tabelas 4 e 5 os casos normais apresentaram sempre uma presença reduzida de ruídos entre as linhas dos harmônicos e também um alcance destes bem alto. Isso demonstra melhor periodicidade da onda, ou seja, uma onda quase periódica durante toda a emissão.

Houve ausência de harmônicos acima de $3,0 \mathrm{kHz}$ em todos os casos de vozes alteradas. Este dado pode ser considerado importante para a avaliação dos espectrogramas de vozes alteradas porque uma onda sonora aperiódica não é amplificada uniformemente, o que impede que seus múltiplos sejam representados no gráfico em forma de linhas harmônicas de acordo com o aumento da freqüência, tornando a região acima de $3,0 \mathrm{kHz}$ escurecida somente pela presença de ruído.

Uma questão interessante a ser considerada é a intensidade de captação das emissões. Para algumas vozes foi necessário modificar o ganho por meio do próprio programa para que se pudesse uniformizar a intensidade das amostras analisadas. Esse procedimento poderia alterar a configuração e intensidade dos harmônicos, que são 
parâmetros importantes na definição da qualidade vocal e na avaliação visual do espectro, mas não modifica o gesto vocal, o que sem dúvida ocorreria na uniformização da intensidade durante a emissão.

Para um próximo estudo sugere-se a manutenção da intensidade de captação para uma análise visual apurada que possa ser melhor relacionada com a análise perceptivoauditiva.

$\mathrm{Na}$ mesma linha de raciocínio, abre-se também a possibilidade da realização de novos estudos que correlacionem essas qualidades vocais com presença, tipo e grau das alterações laríngeas, podendo-se ainda incluir a característica de soprosidade, que não foi abordada neste estudo.

\section{CONCLUSÕES}

Do estudo espectrográfico comparativo dos três tipos de vozes analisadas podemos concluir que:

- Existem diferenças marcantes entre elas, sendo que nas vozes normais existe grande quantidade de harmônicos que alcançam as freqüências mais agudas do espectro, ou seja, acima de 3,0 kHz;

- As vozes ásperas possuem mais harmônicos e melhor definição destes que as roucas e menos que as normais;

- A quantidade de ruído entre as faixas harmônicas é bem menor nas vozes normais, quando comparadas com as vozes ásperas;

- As vozes roucas são as que apresentam maior quantidade de ruídos difusos por todo ou quase todo o espectrograma.

\section{REFERÊNCIAS BIBLIOGRÁFICAS}

1. Baken RJ, Orlikoff RF. Clinical measurement of speech and voice, 2a ed. San Diego: Singular Thomson Learning; 2000. p.225-284.

2. Hirano M. Clinical examination of voice. New York: Springer Verlag; 1981. p.81-4.

3. Mcallister A, Sederholn E, Ternström S, Sundberg J. Perturbation and hoarseness: a pilot study of six children's voices. J Voice 1996;10:252-61

4. Omori K, Kojima H, Kakani R, Slavit DH, Blaugrund SM. Acoustic characteristics of rough voice: subharmonics. J Voice 1997;11:40-7.

5. Rabinov CR, Kreiman J, Gerratt BR, Bielamowicz S. Comparing reliability of perceptual ratings of roughness and acoustic measures of jitter. J Speech Hear Res 1995;38:26-32.

6. Behlau M, Pontes P. Avaliação e tratamento das disfonias. São Paulo: Lovise; 1995. p.97-8.

7. Casper J, Colton R. Compreendendo os problemas da voz: Uma abordagem Fisiológica para o tratamento dos Distúrbios da voz. Porto Alegre: Artes Médicas; 1996.

8. Greene M, Mathieson R. The voice and Its Disorders. $5^{\text {th }}$ ed. London: Wurr; 1989.

9. Moore GP. Voice disorders organically based. In: Travis LE. Handbook of speech Pathology and Audiology. New York: Appleton-Century-Crofts; 1971.

10. Behlau M, Azevedo R, Pontes P. Conceito de voz normal e classificação das disfonias. In: Behlau M. Voz: O livro do especialista. Rio de Janeiro: Revinter; 2001. p.53-79.

11. Hiraoka N, Kitazoe Y, Ueta H, Tanaka S, Tanabe M. Harmonic intensity analysis of normal and hoarse voices. J Acoustic Soc Am 1984;76:1648-51.

12. Behlau M, Tosi O, Pontes P. Determinação da freqüência fundamental e suas variações em altura ("Jitter") e intensidade ("Shimmer"), para falantes do português brasileiro. Acta Awho 1985;4:5-9.

13. Arnold KS, Emanuel FW. Spectral noise levels and roughness severity ratings for vowels produced by male children. J Speech Hear Res 1979;22:613-26.

14. Nunez BF, Suarez NC, Munoz P, Baragano RL, Alvarez ZM, Martinez FA. Estudio espectrografico de la disfonia: subharmonicos. Otorrinolaringol Esp 2000;51:52-6.

15. Deal RE, Emanuel FW. Some waveform and spectral features of vowel roughness. J Speech Hear Res 1978;21:250-64.

16. Emanuel FW, Austin D. Identification of normal and abnormally rough vowels by spectral noise level measurements. J Commun Disord 1981;14:75-85. 